\title{
Multi Hop Routing in Wireless Mobile Networks using Ant Colony Optimization
}

\author{
Harminder Kaur \\ Student \\ Department of Electronics \\ Technology, Guru Nanak Dev \\ University, Amritsar, Punjab,
} India

\author{
Ravinder Singh Sawhney \\ Sr.Lecturer \\ Department of Electronics \\ Technology, Guru Nanak Dev \\ University, Amritsar, Punjab,
}

\author{
Rajan Vohra \\ Assistant Professor \\ Department of Electronics \\ Technology, Guru Nanak Dev \\ University, Amritsar, Punjab,
}

\begin{abstract}
Quality of Service is an indispensable parameter to evaluate the performance of any network, so research engrosseson valuing some important QoS parameters for mobile Adhoc network using AntHocNet routing algorithm. MATLAB software version R2011b is used for simulations due to its ease of node deployment and network set up. Simulation is done while varying the number of packets sent as 10, 30, 50, 70 and 90in a network scenario. It is analyzed that AntHocNet provides excellent performance for fewer hops 1,2 and 3 whereas for increased number of hops 9 and 10, it reveals from the routing metrics that use of AntHocNet is more advantageous over the other routing protocols like DSDV, AODV, DSR and TORA.The simulation enactment graphs shows better performance in terms of End-to-end delay, Delay jitter and superlative performance in terms of SINR with extended number of hops when the payload on the source node increases.
\end{abstract}

\section{General Terms}

MANET, Ant Colony Optimization, Hybrid Algorithm.

\section{Keywords}

AntHocNet,Mobile adhoc network, End-to-end delay, Delay Jitter, SINR

\section{INTRODUCTION}

Since a few years researcher's interest in MANEThas been growing to evolve innovative and efficient ideas in this field.Especially the design of MANET routing protocols has received a lot of attention [1-2]. One of the reasons is that routing in MANET is particularly challenging task. Due to the dynamic topology of the network, efficient paths quickly become inefficient or even infeasible [3-4]. Moreover as the bandwidth of the wireless medium is very limited, so control information flow in the network is very restricted [5-6]. Since there is no infrastructure support for MANET and a destination node may be out of range of a source node. So, a routing procedure is always needed to find an optimal path to forward the packets between source and destination.Numerous routing protocols have been recommended in the existing literature. Some of these like DSDV, AODV, DSR, and TORA have been extensively simulated for various scenarios [7-10].
Ant colony optimization (ACO) is an optimization technique inspired by the exploratory behavior of ants while finding food

[11-12].The routing algorithm based on ants was developed by G. Di Carlo and M. Dorigo [13] and M. Gunes, U. Sorges and I.

Bouazizi in [14] and further discussed in [15]. In this paper, Ant colony optimization (ACO) based metaheuristic is used for optimum route innovation in wireless networks, which has taken aclue from Ant Colony Optimization (ACO) algorithm [16-18].It belongs to class of constructive algorithms that reflect the cooperative behavior of real ants to different optimization problems. The main purpose of the paper is to enrich the performance of routing parameters for mobile ad hoc networks using AntHocNet [19-21] routing algorithm.

\section{SIMULATION ALGORITHM}

The algorithm proposed in this paper AntHocNet has been simulated using the MATLAB software version R2011b with 802.11 MAC protocol as wireless channel.MATLAB is a software package for high performance numerical computation and visualization. It provides an interactive environment with hundreds of built in functions for technical computation, graphics and animation. AntHocNet is a hybrid algorithm works in two phases; reactive path setup and proactive path maintenance and exploration [19-21].

\subsection{Theory for Reactive Path Setup}

The route exploration procedure is started, whenever a source node wants a communication session to the destination node. The source nodebroadcasts reactive forward antsimilar to route request packets of mobile adhoc network routing protocol in order to probe paths to the destination. When the reactive forward ant is received by an intermediary node, it checks whether it has any routing information to the destination in its routing table [19]. If the forward ant has routing information to the destination then intermediary node unicast the reactive forward ant otherwise, it broadcast the reactive forward ant. Due to this broadcasting the reactive forward ants quickly multiply in the network and increase the chances of receipt of duplicate ants.At the destination only the best generation ant is processed and all the duplicate ants are discarded. The best ant received at destination node ischanged into reactive backward ant. This reactive backward ant set up the path from source to destinationby updating the routing table ofintermediary nodes along its way while goingback to the source [20]. The algorithm used for the reactive path setup is as follows: 
Step 1. Source node demands a communication route to the destination.

Step 2. Source node broadcasts Reactive Forward ant.

Step 3. When reactive forward ant reaches at an intermediary node,

a. If duplicate discard

b. Else goto 4 .

Step 4. Each intermediary node looks for routing information

for destination in forward ant routing table.

a) Ifno routing information available broadcast

reactive forward ant.

b) Ifrouting information available, reactive forward

ant choose its next hop with probability,

$\operatorname{Prob}_{n d}=\left(T_{n d}^{p}\right) / \sum_{q \in N_{q d}^{p}} T_{n d}^{p}(1)$

$N_{q d}^{p}$ is the set of neighbors of node $\mathrm{p}$, providing routing information to reach destination node $d$ through the next hop n.

Step 5. Best reactive forward ant received at destination is converted into reactive backward ant that setup path from source to destination by retracing the list of visited nodes byreactive forward ant.

The reactive backward ant update the intermediary node's routing table by calculating local estimate of time at each node. This estimate is used to calculate estimate of time required for an ant to reach from $\mathrm{p}$ to destination $T_{d}^{p}$ and then in turn source to destination, $T_{d}^{S}$.

$T_{d}^{p}=\sum T_{n+1}^{n}, \quad$ where $\mathrm{p} \leq \mathrm{n}<\mathrm{d}, \mathrm{n} \in$ to list of visited nodes by the forward ant and $\mathrm{d}$ corresponds to destination node.

Through this process when the backward ant reaches at the sourcenode, the full path is setup. Now the source node is ready for communication with destination to send data on the discovered path[21].

\subsection{Proactive Path maintenance and exploration}

To maintain the established paths or to explore new ones,the source node brings into play proactive forward ants to update the information about the currently used paths to the destination.This Proactive mechanism is in the form of hello messages. The hello messages are periodically broadcasted. These are short messages broadcasted every $\boldsymbol{T}_{\boldsymbol{h e l l o}}$ seconds by the nodes (e.g. $\boldsymbol{T}_{\text {hello }}=1$ seconds). If a node q receives hello message from a node $\mathrm{p}, \mathrm{q}$ assumes that $\mathrm{p}$ is its neighbor and expects to receive hello message from $\mathrm{p}$ every $\boldsymbol{T}_{\boldsymbol{h} \text { hello }}$ seconds. If qskips certain number of hello messages from p (e.g. 2 hello messages), it assumes that $p$ is no longer its neighbor. Using these messages nodes has routing information about their immediate neighbours in their routing table. In AntHocNet, each node tries to maintain an updated view of its immediate neighbours at any time. When a node q receives the hello messagefrom node $\mathrm{p}$, it checks if it has an entry for destination over neighbor $\mathrm{p}$ in its routing table. If there is no entry, it is an indication of a possible new path from $q$ to destinationthrough $\mathrm{p}$. If it has entry for destination but over other intermediary node it is hint of potential alternate path to destination [19]. AntHocNet detects link failures by the presence or disappearance of the immediate neighbors. If a neighbor is assumed to have disappeared then all the associated entries of that neighbor are deleted from the routing table to clear path informationwith the link failure notification. If the link failure is detected by the failed transmission of a data packet and no other path is available for this data packet, then the node which detects link failure tries local repair of the path with route repair ant [20-21].The next section provides detailed simulation setup followed by results and discussion.

\section{SIMULATION STEPS}

MATLAB software version R2011b is used for AntHocNet algorithm simulations due to its ease of node deployment and network set up.Matlab's built in functions provide excellent tools for linear algebra computations, data analysis, signal processing, optimization etc. and many other types of scientific computations. With the help of MATLAB criticalanalysis of results is achieved by deploying wireless mobile nodes with random uniform distribution where the position of source and destination are $[10,90]$ and $[90,30]$ respectively.

\subsection{Methodology}

- A service area of dimensions $100 \mathrm{~m} \times 100 \mathrm{~m}$ is chosen for MANET infrastructure. The service has a set of mobile nodes.
Each mobile node communicates with each other.
Each mobile node is moving randomly in some
direction with some mobility rate.

To choose the shortest path an efficient form of AntHocNet algorithm is used in which TTL (time to live) is chosen as a threshold parameter for finding the path.

- Here CBR (Constant Bit Rate) is chosen as the traffic type.

- $\quad$ To run the simulation, 10 out of 50 mobile nodes are chosen randomly while the payload on source node is increased.

Table 1 briefly mentions the various simulation parameters and figure 1 shows the simulation scenario in MATLAB.

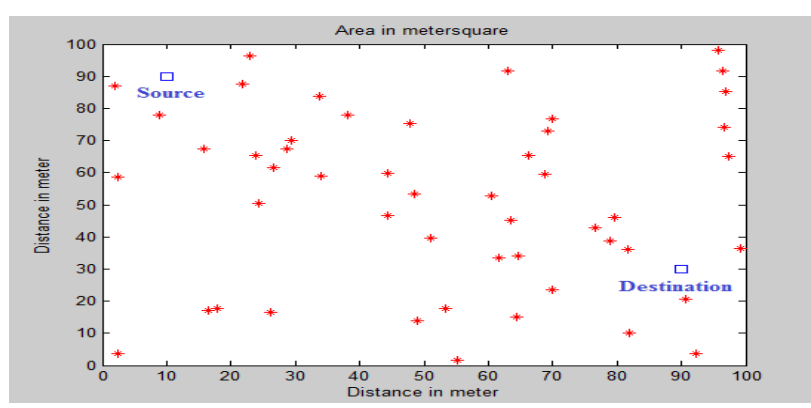

Fig 1: Simulation Scenario 
Table 1. List of Simulation Parameters

\begin{tabular}{|l|l|l|}
\hline S.No. & Parameter & Value \\
\hline 1 & Geographical Area & $100 \times 100 \mathrm{~m}^{2}$ \\
\hline 2 & $\begin{array}{l}\text { Number of Mobile } \\
\text { nodes }\end{array}$ & $\begin{array}{l}\text { Randomly Chosen 10,out of } \\
50\end{array}$ \\
\hline 3 & Routing algorithm & AntHocNet \\
\hline 4 & Mobility model & Random waypoint \\
\hline 5 & $\begin{array}{l}\text { MAC layer } \\
\text { Protocol }\end{array}$ & IEEE 802.11 DCF protocol \\
\hline 6 & Packets Sent & $10,30,50,70,90$ \\
\hline 7 & Traffic Type & CBR \\
\hline 8 & $\begin{array}{l}\text { Performance } \\
\text { parameter }\end{array}$ & $\begin{array}{l}\text { End-to-end delay, Delay jitter } \\
\text { and SINR }\end{array}$ \\
\hline
\end{tabular}

\subsection{Routing Metrics}

\subsubsection{End-To-End Delay}

This is the cumulative statistical measure of the delays experienced by the packets travelling between source and destination and calculated by using,

$$
\begin{array}{r}
\operatorname{Cost}_{p}^{q}=\left(Q_{m a c}^{p}+1\right) D_{m a c}^{p} \\
D_{m a c}^{p} \leftarrow \varepsilon D_{m a c}^{p}+(1-\varepsilon) t_{m a c}^{p}, \varepsilon \in[0,1]
\end{array}
$$

where

$\operatorname{Cost}_{p}^{q}$ is the cost metric when following the wireless link from $p$ to its neighbor $q$.

$D_{m a c}^{p}$ is the expected delay incurred by a data packet when following the wireless link from $p$ to its neighbor $q$.

$Q_{m a c}^{p}$ is the number of packets to be sent that are currently in queue at the MAC layer.

$t_{m a c}^{p}$ is, calculated as a moving average of the time elapsed between the arrival of a packet at the MAC layer and the end of a successful transmission. It is indicated by an acknowledgement received from the next hop.

$\varepsilon$ is a parameter defining how quickly the moving average adapts to new observations (is kept on 0.7).

\subsubsection{Packet Delay Variance (Jitter)}

Jitter could be termed as the variation in delay or packet delay variation. Measuring jitter is critical element to determine the performance of network and the QoS offered by the network. Jitter is commonly used as an indicator of consistency and stability of a network. The jitter is calculated by the equation below:

$$
\text { jitter }=\sum_{p=2}^{k}\left|\left(T_{p}-T_{p-1}\right)-\left(T_{p-1}-T_{p-2}\right)\right|
$$

where

$T_{p}$ is the time of arrival of the $p^{\text {th }}$ packet.

$k$ is the total number of packets received by a destination during a communication session.

\subsubsection{Signal-To-Interference-And-Noise Ratio}

Signal-to-Interference-and-noise ratio is defined as power ratio between meaningful information and the background noise.

$$
\operatorname{Cost}_{p}^{q}=\left\{\begin{array}{ll}
1, & S I N R>S I N R_{t h} \\
\text { constant }>1, & S I N R \leq \operatorname{SINR}_{t h}
\end{array}\right\}
$$

When using SINR to delineateCost $t_{p}^{q}$, AntHocNet does not engrossed about finevariations in the SINR level, but rather in a coarse grained distinction between thelinks on which the probability of successful reception is high and those on which it is low. Therefore,AntHocNet considers a simple approach using a critical SINR value.SINR $R_{t h}$ as threshold, onwhich links with SINR value lower than $S I N R_{t h}$ are penalized.

The cost valueCost ${ }_{p}^{d}$ is inverted, to calculate the pheromone value, using the moving average formula of equation given below:

$$
\sigma_{p q}^{d} \leftarrow \lambda \sigma_{p q}^{d}+[(1-\lambda)] \operatorname{inv}\left(\operatorname{Cost}_{p}^{d}\right), \quad \lambda \in[0,1]
$$

In thisequation $\lambda$ is a parameter regulating the speed of adaptation of the pheromoneto new cost value. In the research, $\lambda$ is taken as 0.7 . The cost value $\operatorname{Cost}_{p}^{d}$ is inverted to calculate the pheromone value $\sigma_{p q}^{d}$, as pheromone indicates thegoodness of a route, rather than its cost.

\section{RESULTS AND DISCUSSIONS}

\subsection{End-to-End delay}

End-to-end delay is measured with respect tovarying payload as shown in figure 2. It is investigated that for few packets i.e. for 10 to 30 packets, the end-to-end delays possess a very small increase up to 3 hops path. When the number of hops proliferates to 9 or 10, again the delaysupsurges by a small amount.Owing to the ability of the algorithm to take stochastic verdicts to route the data packets, ability to detect link failures either with local path repair or by warning preceding nodes on the paths.

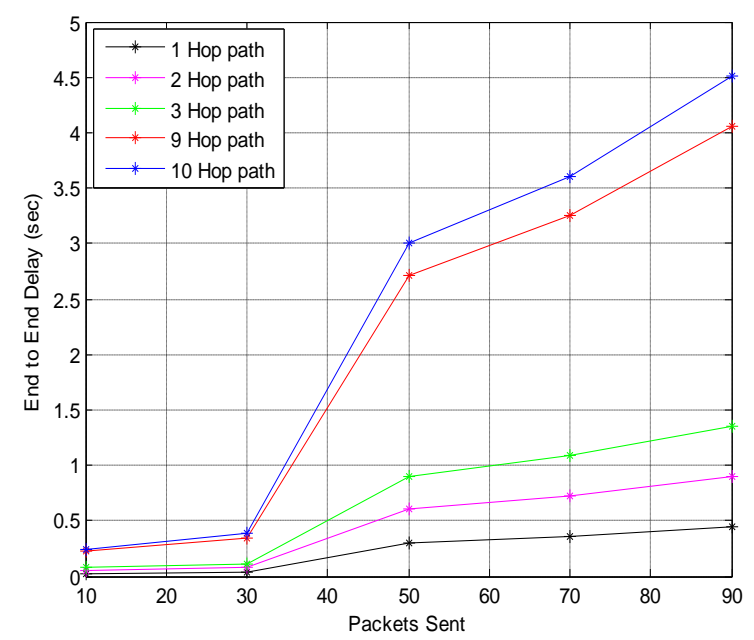

Fig 2: End-to-End delay vs. Number of Packets Sent

It is observed that as the number of packets sent increases from 30 to 50, delays upsurges linearly for the less number of hops. These linear upsurges become steeper with extended number of hops, owing to subsequent path repairing and new path exploration to avoid congestion and wireless channel 
contention is also higher. So packets take more time to trace the network. From the figure 2, it is clear that for 10 hops path, there is difference of 2.618 seconds in end-to-end delay as the packets sent are increased from 30 to 50 whereas for the 3 hop path this difference is 0.7875 seconds.

For higher number of packets from 50 to 90 , difference in end-to-end delay becomes 1.5 seconds for the 10 hops path and 0.45 seconds for the 3 hops path. Because here the system starts to saturate and becomes unable to serve any more packets.

Therefore, with the enactment of AntHocNet algorithm in the real world networks, it would be possible to saturate link delay, when the load on the network is more. Whereas in utmost protocols like DSDV, AODV, DSR and TORA delays upsurges as the load on the network increase.

\subsection{Packet Delay Variance(Jitter)}

Figure 3 demonstratesthe graph between jitter and number of packets sent. It is investigated that the value of jitter obtained is small amongst other routingprotocols like DSDV, AODV, DSR and TORA. It is due to the ability of the AntHocNet routing algorithm to temporary buffer data packets, if no good path between source and destination is available.

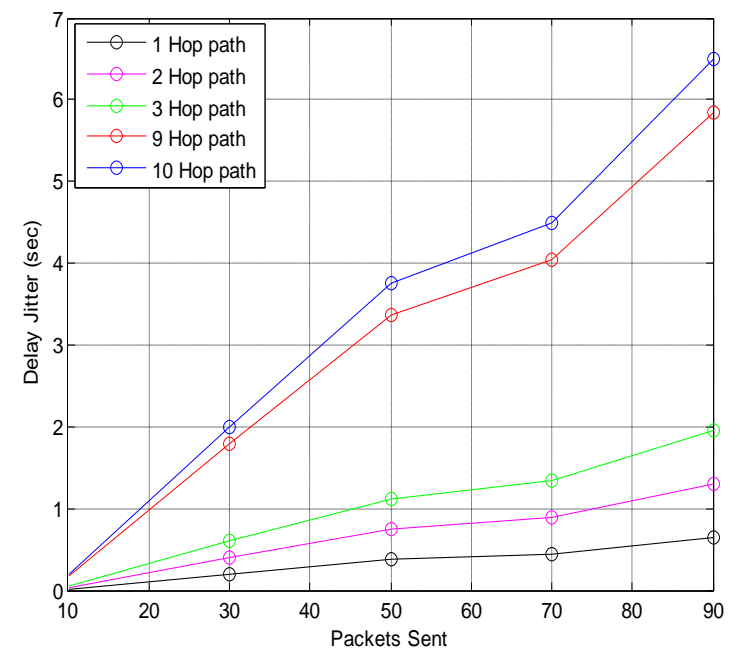

Fig 3: Delay Jitter vs. Number of Packets Sent

From the figure 3 , it is examined that jitter upsurges linearly with the proliferation of packets from 10 to 50, rate of increase is sharp for the extended number of hops in comparison to few hops. This is due to the ability of AntHocNet routing algorithm, proactive route maintenance extends the lifetime of existing paths. As the assumed network scenario considers an omnidirectional antenna as a wireless interface. So the data traffic for all succeeding nodes needs to go over the same outgoing queue and experiencing the delays of other adjacent nodes also. Proceeding further, from the figure 3 it is clear that for the 3 hop path jitter upswings from 0.06 seconds to 1.125 seconds up to 50 packets and total difference of 1.065 seconds is observed, while it is 3.55 seconds for the 10 hop path.

From 50 to 70 packets these upsurges become somewhat stable, sinceat this time network approaches saturation withincreased number of packets. But with further proliferation in packets from 70 to 90 , upsurges in delay becomes steeper for extended hops because each bidirectional link used in the algorithm suffer conflicts due to high load of data packets and the control packets on that particular link.

\subsection{Signal-to-interference-and-noise ratio (SINR)}

SINR is the well-known parameter for appraising the optimal data route, but the methodologies for measuring SINR are inadequate as the evidencesobtained at the physical layer are not sufficient. SINR surges withproliferating packets as algorithm has the ability to detect bad links and avoids them.

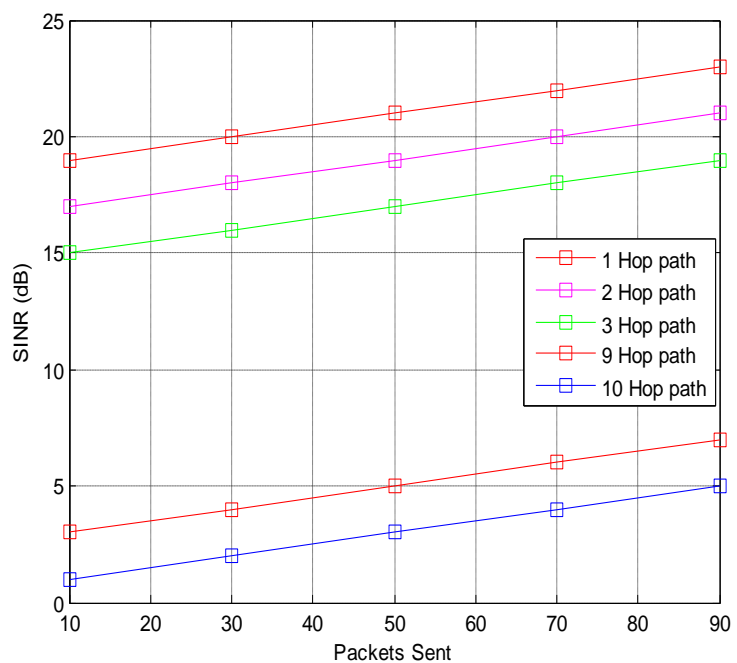

Fig 4: SINR vs. Number of Packets Sent

From figure 4, it is clear thatthere occurs an inverse relation between number of hops and SINR, whereas attenuation on the path increases as the distance traveled increase. It is alsodepicted from figure 4 that for each hop path,risein SINR is constant with value of $4 \mathrm{~dB}$. For 1 hop path SINRupsurgesfrom $19 \mathrm{~dB}$ to $23 \mathrm{~dB}$ with improvement of $4 \mathrm{~dB}$ and for the 10 hop path SINR upsurges from $1 \mathrm{~dB}$ to $5 \mathrm{~dB}$ again with improvement of $4 \mathrm{~dB}$.As the simulation algorithm presumed constant cost value $=1$, for allthe considered paths whose SINR for a specific path comes out to be more than the assumed threshold value.

\section{CONCLUSION}

Simulation results validate the scalability of AntHocNet parallelto routing protocols like DSDV, AODV, DSR and TORA.It is concluded that AntHocNet presents outstandingresults in terms of SINR.It is the best quality of service parameter to regulate the performance of mobile ad hoc network. Simulation results shows improvement of $4 \mathrm{~dB}$ for a particular path who's SINR comes out to be more than the assumed threshold value withthe extended number of hops.In context of end-to-end delay AntHocNet achievesimproved performanceby saturating the link delay for the extended number of hops while the payload at the source node increases from 50 to 90 packets. Because, here the system becomes unable to serve any more packets. In terms of delay jitter, initially jitter increases linearly for few hops and becomes steeper for extended number of hops because the proactive route maintenance of the AntHocNet routing algorithm extends the lifetime of existing paths. With the proliferation of packets from50 to 70, these upsurges become somewhat stable, since at this time network approaches saturation with increased payload. But with further 
proliferation of packets from 70 to 90 , upsurges in delay becomes again steeper for the extended number of hops. Becausehere the bidirectional links used in the algorithm suffer conflicts due to high load of data packets and the control packets.These conflicts canbe reduced by implementing a priority concept at the node level where important packets can be sent first followed by the rest of the packets. This improvement may add up the scalability of the AntHocNet routing algorithm to make its performance even better.

\section{REFERENCES}

[1] L. Wang, Y.T. Shu, O.W.W. Yang, M. Dong, and L.F. Zhang, "Adaptive multipath source routing in wireless ad hoc networks", In Proc. of the IEEE Int. Conf. on Communications, 2001.

[2] M. Elizabeth, and T. Chai-Keong, "A Review of Current Routing Protocols for Ad Hoc Mobile Wireless Networks", IEEE Personal Communications, 1999.

[3] J. Broch, D. A. Maltz, D. B. Johnson.-C. Hu, and J. Jetcheva,"A performance comparison of multi-hop wireless ad hoc network routing protocols" In Proceedings of the Fourth Annual ACM/IEEE International Conference on Mobile Computing and Networking (MobiCom), 1998.

[4] P Jacquet, P Muhlethaler, T Clausen, A Laouiti, A Qayyum, L Viennot, "Optimized link state routing protocol for ad hoc networks", in Proceedings of the 5th IEEE International Multi Topic Conference (INMTC 2001), Le Chesnay, France (2001).

[5] Perumalsamy Deepalakshmi and Shanmugasundaram Radhakrishnan, "An ant colony-based multi objective quality of service routing for mobile ad hoc networks", in EURASIP Journal on Wireless Communications and Networking 2011, 2011:153

[6] R. Ramanathan and J. redi, "A Brief Overview of ad Hoc Networks: Challenges and Directions", IEEECommunications Magazine, 2002.

[7] CE Perkins, P Bhagwat, "Highly dynamic destination sequenced distance vector routing (DSDV) for mobile computers", Computer Communication Review,10(4), 234-244 (1994).

[8] Z Liu, MZ Kwiatkowska, C Constantinou, “A biologically inspired QoS routing algorithm for mobile ad hoc networks",Int. Journal of Wireless and Mobile Computing4(2),6475(2010),doi:10.1504/IJWMC.2010.033054.

[9] David B Johnson and David A Maltz,"Dynamic sourcerouting in ad hoc wireless networks", In Imielinski andKorth, editors, Mobile Computing, pages 153-181, Kluwer Academic Publishers, 1996.

[10] Shahram JAMALI, Sajjad JAHANBAKHSH ${ }^{2, " B A-}$ TORA: A Multipath Routing Protocol for MANETs by Inspiration from Bee and Ant Colonies", PRZEGLĄD
ELEKTROTECHNICZNY (Electrical Review), ISSN 0033-2097 R 87 NR 7/2011.

[11]Dorigo, Marco, Gianni Di Caro, and Luca M. Gambardella. "Ant algorithms for discrete optimization" Artificial Life 1999; 5(2):137-172.

[12] T. Stutzle \& M. Dorigo, 2002, An Experimental Study of the Simple Ant Colony.

[13] G Di Caro, M Dorigo, “AntNet: distributed stigmergetic control for communications networks", J. Artif. Intell. Res. 9, 317-365(1998).

[14] M. Gunes, U. Sorges, I. Bouazizi, 2002, "ARA - The Ant-Colony-Based Routing Algorithm for MANETs", April 2002.

[15] Deepak Bansal, Ravinder Singh Sawhney and Ankur Bansal, "Routing Metrics Improvisation in Wireless Mobile Networks Using Ant Colony Optimization" International Conference on Recent Advances and Future Trends in Information Technology (iRAFIT2012) Proceedings published in International Journal of Computer Applications ${ }^{\circledR}$ (IJCA).

[16] D. Subramanian, P. Druschel and J. Chen, 2003, "Ants and Reinforcement Learning: A Case Study in Routing in Dynamic Networks", May 2003.

[17] Deepak Bansal, Ravinder Singh Sawhney, "Performance Evaluation of Ant Colony Optimization in Mobile Ad Hoc Networks (ACONET) and Comparison of Different Ant Systems", $2^{\text {nd }}$ International Symposium on Information and Communication Technology, Hanoi, Vietnam, 13-14 Oct 2011.

[18] Sarbjeet Kaur, Ravinder Singh Sawhney and Rajan Vohra, "MANET Link Performance Parameters using Ant Colony Optimization Approach",International Journal of Computer Applications (IJCA), Volume 47No.8, June 2012.

[19] G.A. Di Caro, F. Ducatelle, andL.M., Gambardella. "AntHocNet: an ant-based hybrid routing algorithm for mobile ad hoc networks",In Proceedings of PPSNVIII, volume 3242 of LNCS, pages 461-470 Springer, 2004. (Best paper award).

[20] G.A. Di Caro, F. Ducatelle, and L.M. Gambardella, "AntHocNet: an adaptive nature-inspired algorithm for routing in mobile ad hoc networks", European Transactions on Telecommunications, 16(5):443-455, 2005.

[21] G.A. Di Caro, F. Ducatelle, and L.M. Gambardella, "Swarm intelligence for routing in mobile ad hoc networks" ,In Proceedings of the IEEE Swarm Intelligence Symposium, pages 76-83, Pasadena, USA, June 2005.2 IEEE Pres. 